\title{
Three dimensional sound field reproduction using multiple circular loudspeaker arrays
}

\author{
Aastha Gupta, Member, IEEE, and Thushara D. Abhayapala*, Senior Member, IEEE
}

\begin{abstract}
Three dimensional (3D) spatial sound field reproduction enables enhanced immersive acoustic experience for a listener. Recreating an arbitrary 3D spatial sound field using a practically realizable array of loudspeakers is a challenging problem in acoustic signal processing. This paper exploits the underlying characteristics of wavefield propagation to devise a strategy for accurate 3D sound field reproduction inside a 3D region of interest with practical array geometries. Specifically we use the properties of the associated Legendre functions and the spherical Hankel functions, which are part of the solution to the wave equation in spherical coordinates, for loudspeaker placement on a set of multiple circular arrays and provide a technique for spherical harmonic mode-selection to control the repoduced sound field. We also analyze the artifacts of spatial aliasing due to the use of discrete loudspeaker arrays in the region of interest. As an illustration, we design a a third order reproduction system to operate at a frequency of $500 \mathbf{H z}$ with 18 loudspeakers arranged in a practically realizable configuration.
\end{abstract}

Index Terms-Sound field reproduction, Spherical harmonics, Circular loudspeaker array, 3D loudspeaker array, Surround sound

\section{INTRODUCTION}

Three dimensional (3D) sound field reproduction systems offer potential for creating immersive acoustic environments where the listener perceives a realistic but virtual replication of a sound field. This can be achieved by controlling the sound field in a defined spatial region of interest using the signals emitted from a set of loudspeakers. Whilst there are a plethora of techniques for sound field reproduction in two dimensional spatial regions, accurate 3D sound field reproduction is still considered a difficult problem unless the loudspeakers are placed on a sphere that enclose the 3D spatial region of interest. In this paper, we present theory and design of 3D sound field reproduction using practically realizable loudspeaker array geometries in standard rooms.

The method of controlling the loudspeaker signals has been studied under various sound reproduction techniques. Generally, this involves issues relating to spatial loudspeaker placement and calculation of their driving signals for accurate sound reproduction. Arguably there are two main methods of sound field reproduction. A few authors have tried to differentiate the techniques, and understand their similarities however there is much clarity still to be achieved [1]. The first one is based on circular harmonic representation of sound fields and was first introduced in 1973 by Gerzon [2]. The method introduced by Gerzon, is called Ambisonics and

Authors are with the Applied Signal Processing Group, School of Engineering, CECS, The Australian National University. e-mail: Aastha.Gupta@rsise.anu.edu.au, Thushara.Abhayapala@anu.edu.au. mainly focusses on lower order, 2D sound reproduction [3][5]. It has since been further developed into higher order Ambisonics [6]-[9] extensively. In addition there are also some other systems that employ spherical harmonics [10]-[14] for 2D sound reproduction.

The second approach of sound reproduction relies on the Kirchhoff-Helmholtz integral which shows that reproduction is possible inside a given region if the pressure and normal velocity on the surface are known. This is the basis of Wave Field Synthesis (WFS) and was introduced by Berkhout in 1993 [15]. To make this technique more practically applicable, several simplifications have been introduced, such as the use of monopole sources only instead of both monopole and dipole loudspeakers and the selection of a subset of loudspeakers for reproduction in the direction of an external source. This method requires knowledge of the original source also called the primary source. WFS has been applied for 2D sound reproduction using linear and planar arrays [16] and 2.5D [17]. However, to the knowledge of the authors, there is very limited work in 3D sound field reproduction systems using WFS technologies. WFS systems are generally applied for large areas and hence require a significant number of loudspeakers. A 3D WFS system would thus need an increased number of loudspeakers, which needs to be justified for applied use [18]. In [19], a feasibility study of 3D sound field reproduction at low frequencies was reported by controlling the acoustic pressure measured at the boundary surface of the desired region of reproduction.

There are a few systems developed for 3D reproduction using spherical harmonics/ higher order Ambisonics [9], [20][27]. In [20], Poletti compared mode-matching and simple source methods for 3D sound reproduction. He uses regularisation techniques to reduce reproduction errors. There was no optimum solution applicable for accurate reproduction over a large range of frequencies. It was also shown that the number of loudspeakers rises quadratically with the reproduction frequency.

All of the existing 3D sound field reproduction methods require to place the loudspeakers on a sphere that encapsulate the desired reproduction region. Whilst using spherical harmonics, the natural and obvious geometry of loudspeaker placement is a sphere. In terms of user comfort and practice, this is an unpractical approach and thus has hindered the growth of 3D systems using spherical harmonics. The goal of this work is to design a $3 \mathrm{D}$ sound field reproduction system with a flexible loudspeaker array geometry that can be implemented in real rooms and auditoria.

The rest of the paper is organized as follows. We identify 
the issues to be resolved for 3D sound field reconstruction in Section II, and address these in the rest of the paper. We start by describing existing 3D methods using spherical arrays in Section III, and highlight the practical limitations of these that lead to the development of our method using multiple circular arrays. In Section IV, we develop the theory of multiple circular loudspeaker arrays and analyse the underlying characteristics of wavefield propagation. The main contribution is the novel implementation strategy of selected spherical harmonic modes that enables control of the reproduced sound field through its soundfield coefficients. This enables accuracy and full control of the loudspeaker signals to match the desired sound field in the reproduction region and is described in Section V. We also analyse the effect of the discretization error that provides aliasing in the reproduced signal only outside the region of interest in Section VI. We complete the paper in Section VII by discussing a simulation design example for a third order system and the reproduction error in the $3 \mathrm{D}$ region of sound field reproduction.

\section{PRoblem Formulation}

\section{A. Spherical harmonic analysis of sound fields}

In the spherical coordinates system, an efficient set of basis functions to represent sound fields is given by spherical harmonics, which are orthonormal functions of the elevation angle $\theta$ and the azimuth angle $\phi$. An arbitary sound field within a source-free region can be expressed as a linear combination of these spherical harmonics

$$
S(r, \theta, \phi ; k)=\sum_{n=0}^{\infty} \sum_{m=-n}^{n} \alpha_{n m}(k) j_{n}(k r) \mathcal{P}_{n m}(\cos \theta) E_{m}(\phi)
$$

where $m$ and $n(\geq 0)$ are integers, $\alpha_{n m}(k)$ are the spherical harmonic coefficients of the sound field, $k=2 \pi f / c$ is the wavenumber, $f$ is the frequency, $c$ is the speed of sound, $j_{n}(\cdot)$ are the spherical Bessel functions [28] of order $n, E_{m}(\phi)=$ $(1 / \sqrt{2 \pi}) e^{j m \phi}$ are the normalized exponential functions and

$$
\mathcal{P}_{n m}(\cos \theta)=\sqrt{\frac{2 n+1}{2}} \sqrt{\frac{(n-|m|) !}{(n+|m|) !}} P_{n|m|}(\cos \theta)
$$

are the normalized associated Legendre functions. Note that the normalized exponential functions and the normalized associated Legendre functions form orthonormal basis sets in azimuth $\phi \in[0,2 \pi)$ and elevation $\theta \in[0, \pi]$, respectively.

1) Truncation: Let the sound field reproduction region of interest namely $\Omega$ be a sphere of radius $R_{\mathrm{r}}$. Since we are interested in a finite region $\Omega$, the sound field within $\Omega$ can be expressed approximately by a finite set of coefficients, i.e., a finite number of terms of (1). Thus, for a sound field within $\Omega$, the infinite summation in (1) can be truncated [29] to the upper bound of, $N=\left\lceil k e R_{\mathrm{r}} / 2\right\rceil$. The order of the field is related to the radial spatial component of spherical harmonics, given by the spherical Bessel functions [28], [30]. The order depicted by $n$ starts at the smallest radius of the spherical region $\Omega$ being 0 , and varies till the radius $R$, defined by $N=\left\lceil k e R_{\mathrm{r}} / 2\right\rceil$. The spherical harmonic coefficients also vary over modes $m$ ranging from $-n$ to $n$.

\begin{tabular}{c|c|c|c|c|c}
$m \backslash n$ & 0 & 1 & 2 & $\ldots$ & $N$ \\
\hline$N$ & & & & & $\alpha_{N N}$ \\
$\vdots$ & & & & & \\
2 & & & & & $\vdots$ \\
1 & & $\alpha_{11}$ & $\alpha_{21}$ & & $\vdots$ \\
$m=0$ & $\alpha_{00}$ & $\alpha_{10}$ & $\alpha_{20}$ & $\ldots$ & $\alpha_{N 0}$ \\
-1 & & $\alpha_{1(-1)}$ & $\alpha_{2(-1)}$ & & $\vdots$ \\
-2 & & & $\alpha_{2(-2)}$ & & $\vdots$ \\
$\vdots$ & & & & & $\vdots$ \\
$-N$ & & & & & $\alpha_{N(-N)}$
\end{tabular}

TABLE I: sound field coefficients arranged with order $n$ and degree $m$.

2) sound field coefficients: As we are interested in controlling the sound field restricted inside a region $\Omega$ of a defined radius $R_{\mathrm{r}}$, this places a constraint on the spherical harmonics order of the field and making the reproduced field order limited to the truncation factor $N$. Hence, the total number of coefficients required to describe the desired sound field is given by $(N+1)^{2}$ [10]. If we can control, all these $(N+1)^{2}$ spatial coefficients, we can successfully generate the desired sound field inside the region $\Omega$. For a field order limited to $N$, Table I depicts the growth of the number of coefficients as order $n$ with modes $m$ ranging from $-n$ to $n$.

\section{B. Loudspeaker placement}

The desired sound field is given by $(N+1)^{2}$ sound field coefficients. Therefore, the number of loudspeakers required for reproduction must be equal to or greater than this number. Let the number of loudpseakers be $Q$, where

$$
Q \geqslant(N+1)^{2} \text {. }
$$

The questions that arise now are (i) how to find suitable weights or driving functions for the loudspeakers in order to reproduce the desired sound field, and (ii) what are the optimum and/or practical 3D spatial positioning of these loudspeakers to ensure desired reproduction of sound field.

\section{LOUDSPEAKER DRIVING SignaL/WEIGHTS}

\section{A. Direct Least Squares}

For sound field reproduction inside a spherical region $\Omega$ of radius $R_{\mathrm{r}}$, we need to place loudspeakers outside this region. There are a few strategies for loudspeaker placement in the literature, however, here we briefly describe two of the methods that use spherical harmonics.

The simplest method for loudspeaker placement is to randomly distribute them in space. In that instance, let there be $Q>(N+1)^{2}$ loudspeakers randomly placed outside the region $\Omega$ at locations $\boldsymbol{y}_{q} \equiv\left(r_{q}, \theta_{q}, \phi_{q}\right), q=1, \ldots, Q$. The sound field at a point $\boldsymbol{x} \in \Omega$ due to these loudspeakers is then given by

$$
\widetilde{S}(r, \theta, \phi ; k)=\sum_{q=1}^{Q} w_{q}(k) \frac{e^{i k \| \boldsymbol{y}_{q}-\boldsymbol{x}} \|}{\left\|\boldsymbol{y}_{q}-\boldsymbol{x}\right\|}
$$


where $\boldsymbol{x} \equiv\left(r_{\mathrm{r}}, \theta_{\mathrm{r}}, \phi_{\mathrm{r}}\right)$, and $w_{q}(k)$ are the loudspeaker weights. After loudspeaker placement, the goal is to determine the loudspeaker weights which shall produce the desired sound field in the region of interest. We substitute into (3) the JacobiAnger expansion [31] which is given by

$$
\begin{aligned}
\frac{e^{i k \| \boldsymbol{y}_{q}-\boldsymbol{x}} \|}{\left\|\boldsymbol{y}_{q}-\boldsymbol{x}\right\|}= & 4 \pi i k \sum_{n=0}^{\infty} \sum_{m=-n}^{n} h_{n}^{(1)}\left(k y_{q}\right) \mathcal{P}_{n m}\left(\cos \theta_{q}\right) \\
& \times E_{-m}\left(\phi_{q}\right) j_{n}\left(k r_{\mathbf{r}}\right) \mathcal{P}_{n m}\left(\cos \theta_{\mathbf{r}}\right) E_{m}\left(\phi_{\mathbf{r}}\right)
\end{aligned}
$$

where $h_{n}^{(1)}(\cdot)$ is the spherical hankel function, and then equate with (1) to obtain the loudspeaker weights in terms of the sound field coefficients

$$
\alpha_{n m}(k)=4 \pi i k \sum_{q=1}^{Q} w_{q}(k) h_{n}^{(1)}\left(k r_{q}\right) \mathcal{P}_{n m}\left(\cos \theta_{q}\right) E_{-m}\left(\phi_{q}\right) \text {. }
$$

In the above equation, when we substitute the desired sound field's coefficient into $\alpha_{n m}(k)$, we have one known coefficient and $Q$ unknown loudspeaker weights. In order to solve such a system, and estimate all the loudspeaker weights $w_{q}(k)$, we can form a system of simultaneous equations [10] by evaluating (5) for $n=0, \ldots, N$, and $m=-n, \ldots, n$. Such a system of equations could be solved using the least squares method. In that case, the accuracy of sound field reproduction is doubtful, as the method does not study the underlying wavefield complexities and does a brute force approximation to find the weights. So, as there is no control on loudspeaker placement, the rendering thus also has practical limitations, such as not knowing where to place the loudspeakers for varying system environments. In addition, with random loudspeaker placement, it would be difficult to quantify spatial aliasing or sampling issues. In order to have a deeper grasp on the reproduced sound field, a better system is required that considers the underlying wavefield components and reproduction accuracy.

\section{B. Mode-matching on a sphere}

For the analysis of a sound field using spherical harmonics, the ideal geometry for the loudspeaker array due to spatial symmetry would be a sphere [20]. Therefore, a suitable design strategy for sound rendering using spherical harmonics would be to place the loudspeakers on a sphere.

In this solution for sound field reconstruction, a large number of loudspeakers are placed on a sphere with radius $R>R_{\mathrm{r}}$. The loudspeaker driving signals are represented by a spatial function called a loudspeaker aperture function $\rho(\theta, \phi ; k)$, which gives the weights of a loudspeaker positioned at $(\theta, \phi)$ on the sphere of radius $R$. Such a function would describe the spatial-spectral properties of the loudspeakers, and thus enable control over the reproduced sound field. For analytical purposes, consider a continuous aperture function [9] ${ }^{1}$ [20], which is a limiting case of a closely packed set of discrete loudspeakers. The corresponding sound field at a point

\footnotetext{
${ }^{1}$ Also sometimes referred to as loudspeaker aperture or loudspeaker driving function.
}

$\boldsymbol{x} \equiv\left(r_{\mathrm{r}}, \theta_{\mathrm{r}}, \phi_{\mathrm{r}}\right) \in \Omega$ due to such a continuous spherical loudspeaker aperture is given by

$$
S\left(r_{\mathrm{r}}, \theta_{\mathrm{r}}, \phi_{\mathrm{r}} ; k\right)=\int_{0}^{2 \pi} \int_{0}^{\pi} \rho(\theta, \phi ; k) \frac{e^{i(k\|\boldsymbol{y}-\boldsymbol{x}\|)}}{\|\boldsymbol{y}-\boldsymbol{x}\|} \sin \theta d \phi d \theta .
$$

Since the spherical harmonics form a better complete basis set over the unit sphere, we can use them to define the loudspeaker aperture function at a point $(\theta, \phi)$ on a spherical surface. Thus, an arbitrary spherical aperture function can be written using spherical harmonics as

$$
\rho(\theta, \phi ; k)=\sum_{n=0}^{\infty} \sum_{m=-n}^{n} \gamma_{n m}(k) \mathcal{P}_{n m}(\cos \theta) E_{m}(\phi)
$$

where $\gamma_{n m}(k)$ are the spherical harmonic coefficients of the aperture function. By substituting (4) and (7) into (6) and evaluating the integral, we have

$$
\begin{aligned}
S\left(r_{\mathrm{r}}, \theta_{\mathrm{r}}, \phi_{\mathrm{r}} ; k\right)=4 \pi i k \sum_{n=0}^{\infty} & \sum_{m=-n}^{n} \gamma_{n m}(k) h_{n}^{(1)}(k R) \\
& \times j_{n}\left(k r_{\mathrm{r}}\right) \mathcal{P}_{n m}\left(\theta_{\mathrm{r}}\right) E_{m}\left(\phi_{\mathrm{r}}\right) .
\end{aligned}
$$

By equating the loudspeaker sound field (8) with the desired field (1), we can obtain the unknown aperture function coefficients, $\gamma_{n m}(k)$ in terms of the desired sound field coefficients $\alpha_{n m}^{\mathrm{d}}(k)$ as

$$
\alpha_{n m}^{\mathrm{d}}(k)=4 \pi i k \gamma_{n m}(k) h_{n}^{(1)}(k R) .
$$

This method is given in [20] and is called mode-matching since a mode of the desired sound field is matched to the corresponding mode of the aperture function. However, there is a limitation of this method. In practice, the continuous spherical aperture $\rho(\theta, \phi ; k)$ needs to be sampled to find an equivalent array of loudspeakers, and placing loudspeakers equidistantly on a sphere is not straightforward ${ }^{2}$. Similarly, it is hard to imagine having a practical spherical shell loudspeaker array where the desired region of interest is in the middle of the spherical array. Such practical limitations motivate us to consider alternative design geomertries for sound field reproduction using spherical harmonics.

\section{Multiple Circular Array Configuration}

\section{A. Circular aperture}

Consider a circular loudspeaker aperture located at a constant elevation angle $\theta_{q}$ and radial distance $r_{q}$ from the origin. Thus, the aperture function is spatially only dependent on the azimuth angle $\phi$ and let it be depicted by $\rho_{q}(\phi ; k)$. We can represent the circular continuous loudspeaker aperture function by a Fourier series as

$$
\rho_{q}(\phi ; k)=\sum_{m=-\infty}^{\infty} \beta_{m}^{(q)}(k) E_{m}(\phi)
$$

\footnotetext{
${ }^{2}$ However, there are number of possible geometries reported in spherical microphone array literature including vertices of polyhedra.
} 
where $\beta_{m}^{(q)}(k)$ are the frequency dependent Fourier coefficients of the circular aperture function and are given by

$$
\beta_{m}^{(q)}(k)=\int_{0}^{2 \pi} \rho_{q}(\phi ; k) E_{-m}(\phi) d \phi
$$

Now, the resulting sound field due to this circular loudspeaker aperture inside the reproduction area $\Omega$ is

$$
S_{q}\left(r_{\mathrm{r}}, \theta_{\mathrm{r}}, \phi_{\mathrm{r}} ; k\right)=\int_{0}^{2 \pi} \rho_{q}(\phi ; k) \frac{e^{i k\left\|\boldsymbol{y}_{q}-\boldsymbol{x}\right\|}}{\left\|\boldsymbol{y}_{q}-\boldsymbol{x}\right\|} d \phi
$$

where $\boldsymbol{y}_{q} \equiv\left(r_{q}, \theta_{q}, \phi\right)$ is a point on the circular loudspeaker aperture and $\boldsymbol{x} \equiv\left(r_{\mathrm{r}}, \theta_{\mathrm{r}}, \phi_{\mathrm{r}}\right)$ is any point within the desired region of reproduction. Upon substituting the Jacobi-Anger expansion (4) and the Fourier series expansion of the aperture function (10) into (12), we get

$$
\begin{aligned}
S_{q}\left(r_{\mathrm{r}}, \theta_{\mathrm{r}}, \phi_{\mathrm{r}} ; k\right) & =4 \pi i k \sum_{m^{\prime}=-\infty}^{\infty} \sum_{n=0}^{\infty} \sum_{m=-n}^{n} \beta_{m^{\prime}}^{(q)}(k) h_{n}^{(1)}\left(k r_{q}\right) \\
& \times \mathcal{P}_{n m}\left(\cos \theta_{q}\right) \overbrace{\int_{0}^{2 \pi} E_{m^{\prime}}(\phi) E_{-m}(\phi) d \phi}^{\text {orthonormal }} \\
& \times j_{n}\left(k r_{\mathrm{r}}\right) \mathcal{P}_{n m}\left(\cos \theta_{\mathrm{r}}\right) E_{m}\left(\phi_{\mathrm{r}}\right) .
\end{aligned}
$$

Then, by using the orthonormality of the exponential functions, we express (13) in the spherical harmonic expansion form (1) as

$$
\begin{aligned}
S_{q}\left(r_{\mathrm{r}}, \theta_{\mathrm{r}}, \phi_{\mathrm{r}} ; k\right) & =\sum_{n=0}^{\infty} \sum_{m=-n}^{n} \underbrace{4 \pi i k h_{n}^{(1)}\left(k r_{q}\right) \mathcal{P}_{n m}\left(\cos \theta_{q}\right) \beta_{m}^{(q)}(k)}_{\text {coefficients }} \\
& \times j_{n}\left(k r_{\mathrm{r}}\right) \mathcal{P}_{n m}\left(\cos \theta_{\mathrm{r}}\right) E_{m}\left(\phi_{\mathrm{r}}\right) .
\end{aligned}
$$

Therefore, the spherical harmonic coefficients of the sound field in the region of interest $\Omega$ due to a horizontal continuous circular loudspeaker aperture at $\left(r_{q}, \theta_{q}\right)$ with aperture function $\rho_{q}(\phi ; k)$ are given by

$$
\alpha_{n m}^{(q)}(k)=4 \pi i k h_{n}^{(1)}\left(k r_{q}\right) \mathcal{P}_{n m}\left(\cos \theta_{q}\right) \beta_{m}^{(q)}(k)
$$

for $n=-N \cdots N$ and $m=-n \cdots n$.

In order to control the reproduced sound field and hence its spherical harmonic coeffcients, we need to control the right hand side of (15). Upon observing the underlying functions, we see that the normalized associated Legendre functions $\mathcal{P}_{n m}(\cdot)$ have a number of zeros (see Figures 2 and 3 ). Thus, for some values of $n, m$ and $\theta_{q}$, the induced sound field coefficient $\alpha_{n m}^{(q)}(k)$ in (15) is equal to zero irrespective of the value of $\beta_{m}^{(q)}(k)$. We exploit this fact later in the paper to create a design strategy for loudspeaker layouts.

Additionally, a single circular aperture (10) on a circle can only control the sound field coefficients $\alpha_{n m}(k)$ along different degrees $m$ but not on orders $n$ (see (15)). Therefore, we need to consider multiple circular continuous loudspeakers to evaluate aperture coefficients for all desired spherical harmonic coefficients and control the entire reproduced sound field inside $\Omega$.

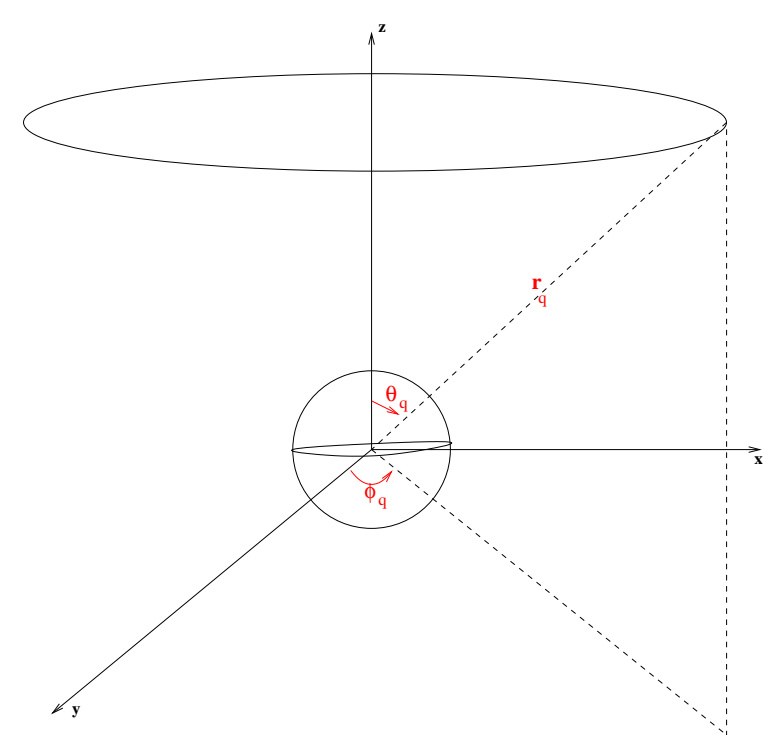

Fig. 1: Circular continuous aperture located at $\left(r_{q}, \theta_{q}\right)$ outside the region of interest $\Omega$



Fig. 2: Magnitude of the normalized associated Legendre functions $\mathcal{P}_{n m}(\cos \theta)$ in $\mathrm{dB}$, where the addition of order $n$ and mode $m$ are even: $(n,|m|)=(0,0) ;(2,0) ;(1,1) ;(2,2) ;(3,1)$.

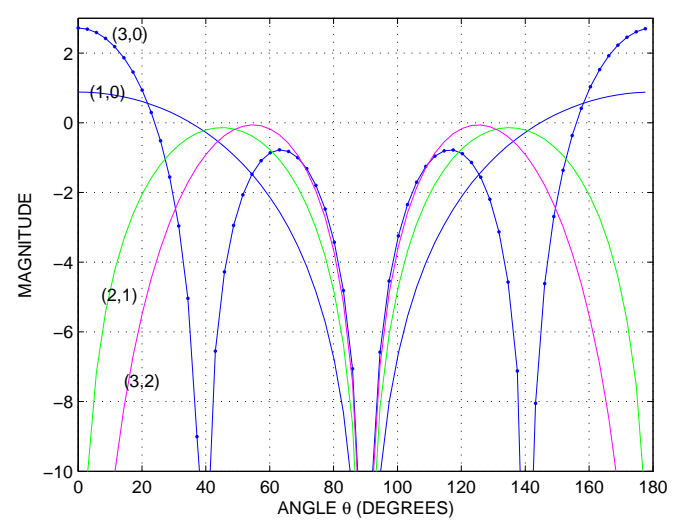

Fig. 3: Magnitude of the normalized associated Legendre functions $\mathcal{P}_{n m}(\cos \theta)$ in $\mathrm{dB}$, where the addition of order $n$ and mode $m$ are odd: $(n,|m|)=(1,0) ;(2,1) ;(3,0) ;(3,2)$. 


\section{B. Multiple circles}

In order to calculate coefficients of all orders and further accurately control the entire reproduced sound field in $\Omega$, we place additional circular loudspeaker apertures in space outside $\Omega$. Suppose there is a set of $Q$ circles of horizontal continuous loudspeakers located at $\left(r_{q}, \theta_{q}\right)$, where $q=1, \ldots, Q$, and the corresponding aperture functions $\rho_{q}(\phi ; k)$ are given by (10). Then the coefficients of the resulting sound field are

$\alpha_{n m}(k)=\sum_{q=1}^{Q} 4 \pi i k h_{n}^{(1)}\left(k r_{q}\right) \mathcal{P}_{n m}\left(\cos \theta_{q}\right) \beta_{m}^{(q)}(k)$, for $n \geqslant m$.

From (16), we note that for a specific $m$, the aperture function coefficients $\beta_{m}^{(q)}(k)$ from all circles contribute to the sound field coefficients of degree $m$ and orders $n \geqslant m$

For a finite dimensional spherical region of interest with radius $R$, the field is truncated to order $N=\lceil k e R / 2\rceil$, and we only need to control sound field coefficients up to order $N$. Thus, by having a sufficient number of circular distributions of loudspeakers, we can control the required countable sound field coefficients to reconstruct a desired given sound field within the region of interest. In the next section, we show how to calculate the aperture function coefficients when given a desired sound field.

\section{Matrix formulation}

Suppose the desired sound field is given by $(N+1)^{2}$ coefficients represented by $\alpha_{n m}^{\mathrm{d}}(k)$. To find the required aperture coefficients $\beta_{m}^{(q)}$, we equate the left hand side of (16) to $\alpha_{n m}^{\mathrm{d}}(k)$ for a specific $m$ and $n=|m|,|m|+1, \cdots, N$ for $Q$ circles. We write the resulting set of simultaneous equations in matrix form, as

$$
\mathbf{A}_{m}=\mathbf{H}_{m} \mathbf{B}_{m}
$$

where $\mathbf{A}_{m}=\left[\alpha_{|m| m}^{\mathrm{d}}(k), \alpha_{(|m|+1) m}^{\mathrm{d}}(k), \ldots, \alpha_{N m}^{\mathrm{d}}(k)\right]^{T}$,

$$
\begin{aligned}
& \mathbf{H}_{m}=4 \pi i k \times \\
& {\left[\begin{array}{ccc}
h_{|m|}^{(1)}\left(k r_{|1|}\right) \mathcal{P}_{m m}\left(\cos \theta_{1}\right) & \cdots & h_{|m|}^{(1)}\left(k r_{Q}\right) \mathcal{P}_{m m}\left(\cos \theta_{Q}\right) \\
\vdots & \ddots & \vdots \\
h_{N}^{(1)}\left(k r_{1}\right) \mathcal{P}_{N m}\left(\cos \theta_{1}\right) & \cdots & h_{N}^{(1)}\left(k r_{Q}\right) \mathcal{P}_{N m}\left(\cos \theta_{Q}\right)
\end{array}\right],}
\end{aligned}
$$

and $\mathbf{B}_{m}=\left[\beta_{m}^{(1)}, \ldots, \beta_{m}^{(Q)}\right]^{T}$.

Equation (17) could be solved for loudspeakers' aperture coefficients $\mathbf{B}_{m}$, using the Least Squares method provided that $\mathbf{H}_{m}$ is non singular. Such a solution may or may not exist if an arbitrary set of circles is used. In a practical set up, we need to avoid certain spatial placement of loudspeakers due to Legendre nulls, which give no energy for reproduction of certain desired reproduction coefficients and field. In the following section we develop a systematic procedure to set up a circular loudspeaker array system.

\section{Mode Matching on Circles}

\section{A. Location of circles using mode selection}

Here we show an implementation process to calculate the aperture coefficients $\beta_{m}^{(q)}(k)$ from different circles, by picking the modes which are best obtained from the respective circles, and we term this process as mode selection. Such a careful strategy of placement enables improved control over the sound field based on spatial properties of the underlying wavefields as well as to consider any practical constraints imposed by room geometries.

We suggest the following procedure to determine valid locations of circular apertures and also to calculate their relevant aperture coefficients $\beta_{m}^{(q)}(k)$ for driving signals.

Step 1 ( $m=N$ series): Consider (16) and Table I for $m=N$, then the only applicable sound field coefficient for this series is $\alpha_{N N}^{\mathrm{d}}(k)$. Since, the desired region of interest is order limited to $N$, as it is in our case, then there are no lower order coefficients and the higher order components have negligible effect inside this region. Therefore, we have

$$
\alpha_{N N}^{\mathrm{d}}(k)=\sum_{q=1}^{Q} 4 \pi i k h_{N}^{(1)}\left(k r_{q}\right) \mathcal{P}_{N N}\left(\cos \theta_{q}\right) \beta_{N}^{(q)}(k) .
$$

In this case, there is only one sound field coefficient $\alpha_{N N}^{\mathrm{d}}(k)$ to be controlled, and hence, we only need to place a single circle for the loudspeaker aperture function. To determine the spatial placement of the loudspeaker aperture function, we need to find an appropriate radius and elevation angle. Consider (19), in order to calculate $\beta_{N}^{(q)}(k)$ for a desired reproduced field, we need to examine the spherical Hankel functions $h_{N}^{(1)}\left(k r_{q}\right)$ and Legendre functions properties $\mathcal{P}_{N N}\left(\cos \theta_{q}\right)$ for this circle. From Figure 2, we observe that there are certain elevation angles where the magnitude of the field is quite low or zero. These angles are avoided, because at these angles the energy is not enough to provide the desired sound field coefficient $\alpha_{N N}^{\mathrm{d}}(k)$ and hence the resulting reproduced field will not be as accurate as desired. Thus, we choose $\theta_{q}$ such that the associated Legendre functions have significantly large and stable values (see Figure 2). Secondly, as we are considering an even harmonic, thus, we select $\theta_{q}=\pi / 2$, as all even ${ }^{3}$ associated Legendre functions at this angle have good values to ensure accuracy and stability.

Therefore, we choose the $x-y$ plane to place the first circle. We also set $\beta_{N}^{(q)}(k)=0$ for all other circles $q=2 \cdots Q$, which we will add to the system in the subsequent steps. Thus, (19) reduces to

$$
\alpha_{N N}^{\mathrm{d}}(k)=4 \pi i k h_{N}^{(1)}\left(k r_{1}\right) \mathcal{P}_{N N}\left(\cos \theta_{1}\right) \beta_{N}^{(1)}(k),
$$

which can be used to determine the loudspeaker aperture function $\beta_{N}^{(1)}(k)$ coefficient for the $m=N$ series from the first circle as

$$
\beta_{N}^{(1)}(k)=\frac{\alpha_{N N}^{\mathrm{d}}(k)}{4 \pi i k h_{N}^{(1)}\left(k r_{1}\right) \mathcal{P}_{N N}\left(\cos \theta_{1}\right)} .
$$

Step 2 ( $m=N-1$ series): For this series, consider Table I, there are two available desired harmonic coefficients $\alpha_{N-1 N-1}^{\mathrm{d}}(k)$ and $\alpha_{N N-1}^{\mathrm{d}}(k)$. Therefore, we need at least two

\footnotetext{
${ }^{3}$ Even and odd are defined as the sum of the scalar values of order $n$ and degree $m$ are equal to an even integer and odd integer, respectively.
} 
aperture function coefficients $\beta_{N-1}^{(q)}(k), q=1,2$ to be calculated that would render the desired field for the $m=N-1$ series. Since there are two sound field coefficients, the system of simultaneous equations (16) only has two equations. From (17) we have,

$$
\begin{aligned}
& \alpha_{N-1 N-1}^{\mathrm{d}}(k)=\sum_{q=1}^{Q} 4 \pi i k h_{N-1}^{(1)}\left(k r_{q}\right) \mathcal{P}_{N-1 N-1}\left(\cos \theta_{q}\right) \beta_{N-1}^{(q)} \\
& \text { and } \\
& \alpha_{N N-1}^{\mathrm{d}}(k)=\sum_{q=1}^{Q} 4 \pi i k h_{N}^{(1)}\left(k r_{q}\right) \mathcal{P}_{N N-1}\left(\cos \theta_{q}\right) \beta_{N-1}^{(q)}(k) .
\end{aligned}
$$

As we need at least two unknown aperture function coefficients, we require two loudspeaker apertures or circles. In other words, we need $\beta_{N-1}^{(q)}(k)$, from two circles to realize the desired sound field coefficients. As one of the sound field coefficients $\alpha_{N-1 N-1}^{\mathrm{d}}(k)$ is even, we can reuse the first circle $(q=1)$, for the aperture coefficient $\beta_{N-1}^{(1)}(k)$. Now, the next step is to find an appropriate valid placement of the second circle and ensure that the desired spherical harmonic $\alpha_{N N-1}^{\mathrm{d}}(k)$ is present inside the region of interest $\Omega$. The valid choices of $r_{q}$ and $\theta_{q}$ would ensure accurate calculation of $\beta_{N-1}^{(2)}(k)$ for the second loudspeaker aperture.

The second circle needs to be located at a particular elevation angle $\theta_{2}$ where $\mathcal{P}_{N(N-1)}\left(\theta_{2}\right) \neq 0$. We can use Figure 3 to determine an appropriate value ${ }^{4}$ for $\theta_{2}$ such that there is significant energy for the relevant Legendre functions and spherical harmonics. We also set $\beta_{N-1}^{(q)}(k)=0$ for $q>2$, i.e., for other circles. To obtain the aperture coefficients, we solve the system of simultaneous equations or matrix equation (17) which becomes $\mathbf{A}_{N-1}=\mathbf{H}_{N-1} \mathbf{B}_{N-1}$. Thus,

$$
\left[\begin{array}{l}
\beta_{N-1}^{(1)} \\
\beta_{N-1}^{(2)}
\end{array}\right]=4 \pi i k \mathbf{H}_{N-1}^{-1}\left[\begin{array}{c}
\alpha_{(N-1)(N-1)}^{\mathrm{d}}(k) \\
\alpha_{N(N-1)}^{\mathrm{d}}(k)
\end{array}\right]
$$

where $\mathbf{H}_{N-1}^{-1}$ is the inverse of $\mathbf{H}_{N-1}=i k \times$

$$
\left[\begin{array}{cc}
h_{N-1}^{(1)}\left(k r_{1}\right) \mathcal{P}_{N-1 N-1}\left(\cos \theta_{1}\right) & h_{N-1}^{(1)}\left(k r_{2}\right) \mathcal{P}_{N-1 N-1}\left(\cos \theta_{2}\right) \\
h_{N}^{(1)}\left(k r_{1}\right) \mathcal{P}_{N N-1}\left(\cos \theta_{1}\right) & h_{N}^{(1)}\left(k r_{2}\right) \mathcal{P}_{N N-1}\left(\cos \theta_{2}\right)
\end{array}\right] \text { each }
$$

$$
\begin{aligned}
\alpha_{N-1 N-2}^{\mathrm{d}}(k)=\sum_{q=1}^{Q} 4 \pi i k h_{N-1}^{(1)}\left(k r_{q}\right) & \\
& \mathcal{P}_{N-1 N-2}\left(\cos \theta_{q}\right) \beta_{N-2}^{(q)}(k) .
\end{aligned}
$$

and

$$
\alpha_{N N-2}^{\mathrm{d}}(k)=\sum_{q=1}^{Q} 4 \pi i k h_{N}^{(1)}\left(k r_{q}\right) \mathcal{P}_{N N-2}\left(\cos \theta_{q}\right) \beta_{N-2}^{(q)}(k) .
$$

In this case, we have two even and one odd sound field coefficients namely $\alpha_{N-2 N-2}^{\mathrm{d}}(k)$ and $\alpha_{N N-2}^{\mathrm{d}}(k)$. Since the first circle $(q=1)$ is on the $\mathrm{x}-\mathrm{y}$ plane, we reuse it to control the even harmonic coefficient $\alpha_{N-2 N-2}^{\mathrm{d}}(k)$, and the second circle $(q=2)$ to control the odd harmonic $\alpha_{N-1 N-2}^{\mathrm{d}}(k)$. Since there are two even coefficients, we introduce a third circle to control $\alpha_{N N-2}^{\mathrm{d}}(k)$ with appropriate $\theta_{3}$ where $\mathcal{P}_{(N)(N-2)}\left(\theta_{3}\right) \neq 0$. As before, we set $\beta_{N-2}^{(q)}(k)=0$ for $q>3$. Now we use (17) to determine $\beta_{N-2}^{(q)}(k)$ for $q=1,2,3$ as

$$
\left[\begin{array}{l}
\beta_{N-2}^{(1)} \\
\beta_{N-2}^{(2)} \\
\beta_{N-2}^{(3)}
\end{array}\right]=\mathbf{H}_{N-2}^{-1}\left[\begin{array}{c}
\alpha_{(N-2)(N-2)}^{\mathrm{d}}(k) \\
\alpha_{(N-1)(N-2)}^{\mathrm{d}}(k) \\
\alpha_{(N)(N-2)}^{\mathrm{d}}(k)
\end{array}\right]
$$

where $\mathbf{H}_{N-2}^{-1}$ is the inverse of $\mathbf{H}_{N-2}$.

Step N+1 ( $m=0$ series): There are $N+1$ coefficients in this series. Hence, we can reuse all previously established circles together with a new circle. Since, the final circle is needed for a single coefficient, it can be a single point at $\theta_{N+1}=0$. As before, $\beta_{0}^{(q)}(k)$ for $q=1, \ldots, N+1$ can be calculated from (17).

Note that the same set of circles could be reused for negative values of $m$, i.e., starting with $m=-N$ from the first circle.

\section{B. Loudspeaker driving signals}

As a result of the mode-matching algorithm, we have calculated all the aperture coefficients from all the circles, that can be used to evaluate the loudspeaker aperture functions for each respective $q$ th circle by

$$
\rho_{q}(\phi ; k)=\sum_{m=-N_{q}}^{N_{q}} \beta_{m}^{(q)}(k) E_{m}(\phi)
$$

Step 3 ( $m=N-2$ series): In this case, we need to calculate three aperture function coefficients to control three harmonic sound field coefficients $\alpha_{N-2 N-2}^{\mathrm{d}}(k), \alpha_{N-1 N-2}^{\mathrm{d}}(k)$ and $\alpha_{N N-2}^{\mathrm{d}}(k)$. From (17) we have,

$$
\begin{aligned}
\alpha_{N-2 N-2}^{\mathrm{d}}(k)=\sum_{q=1}^{Q} 4 \pi i k & h_{N-2}^{(1)}\left(k r_{q}\right) \\
& \mathcal{P}_{N-2 N-2}\left(\cos \theta_{q}\right) \beta_{N-2}^{(q)}(k),
\end{aligned}
$$

\footnotetext{
${ }^{4} \mathrm{~A}$ complete guideline to choosing elevation angles for even and odd associated Legendre functions are given in [32].
}

where $N_{q}$ is the highest active mode of the $q$ th loudspeaker aperture.

\section{DisCRETIZATION}

\section{A. Sampling of circles}

In order to have a practically realizable loudspeaker array, we cannot have continuous aperture functions. A more suitable representation of the loudspeaker array would be to discretize the continuous aperture functions. Therefore, consider (28), and let the circular aperture be sampled on $L_{q}$ points given by $\ell=1, \cdots, L_{q}$. According to Shannon's theorem for a 
complete representation $L_{q} \geqslant 2 N_{q}+1$. Thus, the weight of the $\ell^{t h}$ loudspeaker located at the $q^{\text {th }}$ circle is given by

$$
\rho_{q}\left(\phi_{q}^{(\ell)} ; k\right)=\frac{2 \pi}{L_{q}} \sum_{m=-N_{q}}^{N_{q}} \beta_{m}^{(q)}(k) E_{m}\left(\phi_{q}^{(\ell)}\right) \text { for } \ell=1 \ldots L_{q}
$$

where the factor $2 \pi / L_{q}$ results from uniform sampling. However, we could use nonuniform sampling as long as the maximum sensor separation angle is less than $2 \pi / N_{q}$. In the next section, we quantify the errors involved with the above discretization in the reproduced field.

\section{B. Sampling Error in reconstruction}

There is an inherent error in reproduction due to discretization of the aperture function and this is a natural byproduct of practical implementation of the sound field reproduction system. Here, we analyse this aliasing error and its effect on the sound reproduction. As it is a linear system, we can objectively say

$$
S_{R}=S_{D}+S_{A}
$$

where $S_{R}$ is the reproduced field in the region of interest, $S_{D}$ the desired sound field and $S_{A}$ is the aliasing error in the reproduced sound field, respectively.

Let the loudspeakers on a given circle be equally spaced and on positions given as $\phi_{q}^{(\ell)}=\left(2 \pi \ell / L_{q}\right)$. Then, the general expression for the reproduced sound field from a discretized loudspeaker array consisting of $Q$ circular arrays (where $q$ th circular array consists of $L_{q}$ loudspeakers) is given by

$$
S_{R}\left(r_{\mathrm{r}}, \theta_{\mathrm{r}}, \phi_{\mathrm{r}} ; k\right)=\sum_{q=1}^{Q} \sum_{\ell=1}^{L_{q}} \frac{2 \pi}{L_{q}} \rho_{q}\left(\phi^{(\ell)}, k\right) \frac{e^{i k\left\|\boldsymbol{y}_{q}^{\ell}-\boldsymbol{x}\right\|}}{\left\|\boldsymbol{y}_{q}^{\ell}-\boldsymbol{x}\right\|}
$$

where $2 \pi / L_{q}$ is the discretization step or the angular loudspeaker spacing on the aperture. Upon substituting for the aperture function $\rho_{q}\left(\phi^{(\ell)}, k\right)$ from (28) and the Jacobi-Anger expansion (4) we obtain

$$
\begin{array}{r}
S_{R}\left(r_{\mathrm{r}}, \theta_{\mathrm{r}}, \phi_{\mathrm{r}} ; k\right)=\sum_{q=1}^{Q} \sum_{\ell=1}^{L_{q}} \sum_{m=-N_{q}}^{N_{q}} \frac{2 \pi}{L_{q}} \beta_{m}^{(q)}(k) E_{m}\left(\phi_{q}^{(\ell)}\right) \\
\times 4 \pi i k \sum_{n=0}^{\infty} \sum_{m^{\prime}=-n}^{n} h_{n}^{(1)}\left(k r_{q}\right) \mathcal{P}_{n m^{\prime}}\left(\cos \theta_{q}\right) E_{-m^{\prime}}\left(\phi_{q}^{(\ell)}\right) \\
\times j_{n}\left(k r_{\mathrm{r}}\right) \mathcal{P}_{n m^{\prime}}\left(\cos \theta_{\mathrm{r}}\right) E_{m^{\prime}}\left(\phi_{\mathrm{r}}\right) .
\end{array}
$$

We rewrite the above expression as

$$
\begin{aligned}
S_{R}\left(r_{\mathrm{r}}, \theta_{\mathrm{r}}, \phi_{\mathrm{r}} ; k\right) & =\sum_{q=1}^{Q} \sum_{n=0}^{\infty} \sum_{m^{\prime}=-n}^{n} \sum_{m=-N_{q}}^{N_{q}} 4 \pi i k \beta_{m}^{(q)}(k) h_{n}^{(1)}\left(k r_{q}\right) \\
& \times \overbrace{\sum_{\ell=1}^{L_{q}} \frac{2 \pi}{L_{q}} E_{m}\left(\phi_{q}^{(\ell)}\right) E_{-m^{\prime}}\left(\phi_{q}^{(\ell)}\right)}^{\text {orthogonal }} \mathcal{P}_{n m^{\prime}}\left(\cos \theta_{q}\right) \\
& \times j_{n}\left(k r_{\mathrm{r}}\right) \mathcal{P}_{n m^{\prime}}\left(\cos \theta_{\mathrm{r}}\right) E_{m^{\prime}}\left(\phi_{\mathrm{r}}\right) .
\end{aligned}
$$

From the geometric progression [33], for $\phi_{q}^{\ell}=\ell 2 \pi / L_{q}$, we have

$$
\sum_{\ell=1}^{L_{q}} E_{m}\left(\phi_{q}^{\ell}\right) E_{-m^{\prime}}\left(\phi_{q}^{\ell}\right)= \begin{cases}\frac{L_{q}}{2 \pi} & \text { if } m^{\prime}=m+s L_{q} \\ 0 & \text { otherwise }\end{cases}
$$

where $s$ is an integer. By substituting (31) into (30), the reconstructed field becomes

$$
\begin{aligned}
& S_{R}\left(r_{\mathrm{r}}, \theta_{\mathrm{r}}, \phi_{\mathrm{r}} ; k\right)=\sum_{s=-\infty}^{\infty} \sum_{n=0}^{\infty} \sum_{m=-n}^{n} \sum_{q=1}^{Q} 4 \pi i k \beta_{m+s L_{q}}^{(q)}(k) \\
& \times h_{n}^{(1)}\left(k r_{q}\right) \mathcal{P}_{n m}\left(\cos \theta_{q}\right) j_{n}\left(k r_{\mathrm{r}}\right) \mathcal{P}_{n m}\left(\cos \theta_{\mathrm{r}}\right) E_{m}\left(\phi_{\mathrm{r}}\right)
\end{aligned}
$$

Note that the $s=0$ term in (32) gives us the desired field, i.e.,

$$
\begin{aligned}
S_{D}\left(r_{\mathrm{r}}, \theta_{\mathrm{r}}, \phi_{\mathrm{r}} ; k\right) & =\sum_{n=0}^{\infty} \sum_{m=-n}^{n} \underbrace{\sum_{q=1}^{Q} 4 \pi i k \beta_{m}^{(q)}(k) h_{n}^{(1)}\left(k r_{q}\right) \mathcal{P}_{n m}\left(\cos \theta_{q}\right)}_{\alpha_{n m}^{\mathrm{d}}(k)} \\
& \times j_{n}\left(k r_{\mathrm{r}}\right) \mathcal{P}_{n m}\left(\cos \theta_{\mathrm{r}}\right) E_{m}\left(\phi_{\mathrm{r}}\right) .
\end{aligned}
$$

All the other terms in (32) for $s \neq 0$ produce undesirable errors. However, we can quantify these errors to see their effect to the sound field reproduced inside the desired region of interest $\Omega$. Thus, the aliasing error is given by

$$
\begin{aligned}
& S_{A}\left(r_{\mathrm{r}}, \theta_{\mathrm{r}}, \phi_{\mathrm{r}} ; k\right)=\sum_{\substack{s=-\infty \\
s \neq 0}}^{\infty} \sum_{n=0}^{\infty} \sum_{m=-n}^{n} \sum_{q=1}^{Q} 4 \pi i k \beta_{m+s L_{q}}^{(q)}(k) \\
& \times h_{n}^{(1)}\left(k r_{q}\right) \mathcal{P}_{n m}\left(\cos \theta_{q}\right) j_{n}\left(k r_{\mathrm{r}}\right) \mathcal{P}_{n m}\left(\cos \theta_{\mathrm{r}}\right) E_{m}\left(\phi_{\mathrm{r}}\right) .
\end{aligned}
$$

We have following comments on the aliasing error:

- Since the desired reproduction region $\Omega$ has a finite dimension of radius $R_{\mathrm{r}}$, the field inside it is mode limited to $N \approx\left\lceil k e R_{\mathrm{r}} / 2\right\rceil$. Hence, the summation $\sum_{n=0}^{\infty}$ is truncated to $N$.

- Also, recall that we only illuminate limited number of modes of each circular loudspeaker array. I.e., we have the following constraint on the loudspeaker modal coefficients $\beta_{m}^{(q)}(k)=0$ for $|m|>N_{q}$. Therefore,

$$
\beta_{\left(m+s L_{q}\right)}^{(q)}(k)=0 \text { for }\left|m+s L_{q}\right|>N_{q} .
$$

Thus, the aliasing error given by (34) is non zero for integer values of $s$ that satisfies the constraint $-N_{q}-m \leq$ $s L_{q} \leq N_{q}-m$. Also note that $|m| \leq N \approx\left\lceil k e R_{\mathrm{r}} / 2\right\rceil$, $L_{q} \geq\left(2 N_{q}+1\right)$, and $N_{q} \leq N$. It can be shown that only $s=0$ satisfies all these constraints. Thus, the aliasing error is zero inside the desired reproduction region for a loudspeaker array design for a particular frequency. However, the aliasing error is non-zero for higher frequencies inside the reproduction region. Also, there is aliasing error outside the desired reproduction region even at the design frequency. One can quantitively calculate these errors for various scenarios using (34). 
This article has been accepted for publication in a future issue of this journal, but has not been fully edited. Content may change prior to final publication.

\section{Simulation}

\section{A. Design Example}

To illustrate the new method, we simulate a third order system $(N=3)$ in this paper. According to the truncation theory $N \approx\left\lceil k e R_{\mathrm{r}} / 2\right\rceil$. Thus, there are three interconnected variables for the reproduction region; order $N$, radius $R_{\mathrm{r}}$ and wave number $k$ or frequency $f$. Based on reasonable practical systems we choose the order to be $N=3$, and frequency of operation $f=500 \mathrm{~Hz}$. In which case, the region of interest is limited to a radius of $R_{\mathrm{r}}=0.11 \mathrm{~m}$. If we consider the size of a typical human head, such a region of interest seems reasonable. Higher orders of the reproduced sound field lie outside the $0.11 \mathrm{~m}$ radius of the region of interest. In this paper, we shall focus our attention to this limited region for accuracy of sound field reproduction. For a larger reproduction region, we would require a higher order system and/or lower frequency of operation.

After setting the desired region of reproduction, we need to carefully select the placement of the circular loudspeaker arrays based on the mode selection process and underlying wavefield properties of the Hankel and Legendre functions as described in Section V. The mode selection method ensures that each circular loudspeaker array controls coefficients upto a certain order $N_{q}$.

Geometry: Following the technique provided in Section V, for this design example, we place the first circular array on the xy plane to control the even spherical harmonics i.e., at elevation angle $\theta_{1}=90^{\circ}$ and radius $r_{1}=1.8 \mathrm{~m}$. The loudspeaker aperture in this circle is mode limited to $N_{1}=N=3$. The next circle is used for controlling the odd spherical harmonics with mode limit of $N_{2}=2$ and, is placed at $\theta_{2}=65^{\circ}$ and radial distance $r_{2}=1.9 \mathrm{~m}$ from the origin. The third circular array controls the even harmonics of lower orders with a mode limit of $N_{3}=1$ and is placed at $\theta_{3}=25^{\circ}$ and radial distance $r_{3}=2 \mathrm{~m}$. The last array is merely a single loudspeaker to control the mode limit $N_{4}=0$ harmonics and is placed on the $\mathrm{z}$-axis, at $\theta_{4}=0^{\circ}$ (above the listening region) and at a radial distance $r_{4}=1.8 \mathrm{~m}$. The radii of the different circles are chosen such that the array can be fit into a typical room with minimum dimensions of $4 \mathrm{~m} \times 4$ $\mathrm{m} \times 3 \mathrm{~m}$.

Note that, for a third order system, there are a total of three circular arrays and a single loudspeaker placed on the $z$-axis above the $x y$ plane and have radii approximately comparable to that of a room, or fit in a cylindrical shape inside a standard room. Based on the truncation limit of each circle, the minimum number of loudspeakers on each circle is given by $2 N_{q}+1=7,5,3,1$. However, we include an extra loudspeaker at the second and third circles to improve the accuracy of the reproduced field. Thus, there are a total of 18 loudspeakers in a multiple circular loudspeaker array configuration to control 16 coefficients. The aperture function coefficients $\beta_{m}^{(q)}(k)$ for each circle is as described in Section V. The design information is tabulated in Table II together with the illuminated aperture coefficients for each circle.

\begin{tabular}{|c|c|c|c|c|}
\hline$q$ & $\left(r_{q}, \theta_{q}\right)$ & $\begin{array}{c}\text { Mode limit } \\
N_{q}\end{array}$ & $\begin{array}{c}L_{q} \\
=2 N_{q}+1\end{array}$ & Aperture modes $\beta_{m}^{(q)}$ \\
\hline 1 & $1.8 \mathrm{~m}, 90^{\circ}$ & 3 & 7 & $\beta_{ \pm 3}^{1}, \beta_{ \pm 2}^{1}, \beta_{ \pm 1}^{1}, \beta_{0}^{1}$ \\
2 & $1.9 \mathrm{~m}, 65^{\circ}$ & 2 & 5 & $\beta_{ \pm 2}^{2}, \beta_{ \pm 1}^{2}, \beta_{0}^{2}$ \\
3 & $2 \mathrm{~m}, 25^{\circ}$ & 1 & 3 & $\beta_{ \pm 1}^{3}, \beta_{0}^{3}$ \\
4 & $1.8 \mathrm{~m}, 0^{\circ}$ & 0 & 1 & $\beta_{0}^{4}$ \\
\hline
\end{tabular}

TABLE II: Design formulation for a third order system using four circles to determine loudspeaker aperture coefficients $\beta_{m}^{(q)}$

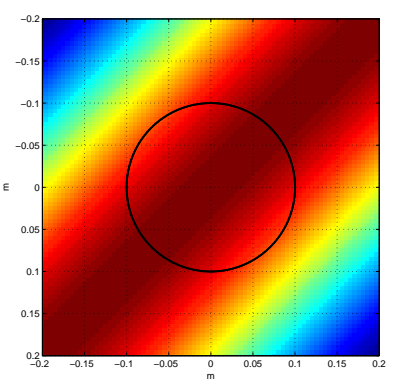

(a)



(c)

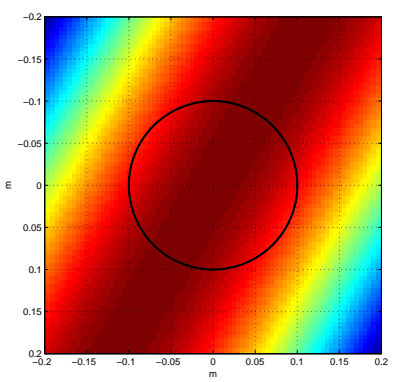

(b)



(d)
Fig. 4: Real part of the desired and reproduced sound fields at an operating frequency of $500 \mathrm{~Hz}$ : (a) Desired sound field corresponding to a plane wave arriving from a direction of $(\theta, \phi)=\left(90^{\circ}, 45^{\circ}\right)$, (b) Desired sound field corresponding to a plane wave arriving from a direction of $(\theta, \phi)=\left(135^{\circ}, 60^{\circ}\right)$, (c) Reproduced sound field corresponding to (a), and (d) Reproduced sound field corresponding to (b).

\section{B. Example sound field}

To demonstrate the designed loudspeaker array, we reproduce a plane wave sound field and observe it in the region of interest. To illustrate, we plot the real part of the reproduced sound field within a square slice of the region of interest on the $x-y$ plane. The desired and reproduced fields are shown in Figure 4, where the encircled region is the region of interest for a third order system and the plane wave is arriving at a direction of $(\theta, \phi)=\left(90^{\circ}, 45^{\circ}\right)$ and $(\theta, \phi)=\left(135^{\circ}, 60^{\circ}\right)$ at an operating frequency of $f=500 \mathrm{~Hz}$. Observe that within the encircled region, the desired and reproduced fields are similar, hence showing accurate reproduction. The root squared error between the desired and the reproduced field of the above two examples are given in Fig. 5a and Fig. 5b respectively. A quantitative error analysis over entire spherical region of interest is given in the next subsection. 


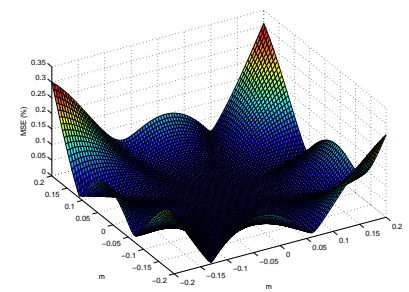

(a)

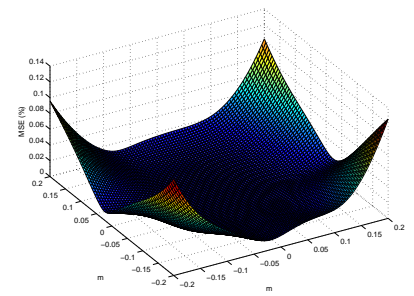

(b)
Fig. 5: Root squared error between the desired and reproduced sound fields at $500 \mathrm{~Hz}$ : (a) a plane wave arriving from a direction of $(\theta, \phi)=\left(90^{\circ}, 45^{\circ}\right)$, (b) a plane wave arriving from a direction of $(\theta, \phi)=\left(135^{\circ}, 60^{\circ}\right)$.

\section{Mean Square Error over 3D}

We define the Mean Square Error (MSE) of the reproduction over the spherical reproduction region of radius $R_{\mathrm{r}}$ as

$\mathrm{MSE}=\sqrt{\frac{\int_{0}^{R_{\mathrm{r}}} \int_{0}^{2 \pi} \int_{0}^{\pi}\left|S_{R}\left(r_{\mathrm{r}}, \theta_{\mathrm{r}}, \phi_{\mathrm{r}} ; k\right)-S_{D}\left(r_{\mathrm{r}}, \theta_{\mathrm{r}}, \phi_{\mathrm{r}} ; k\right)\right|^{2} d \Omega}{\int_{0}^{R_{\mathrm{r}}} \int_{0}^{2 \pi} \int_{0}^{\pi}\left|S_{D}\left(r_{\mathrm{r}}, \theta_{\mathrm{r}}, \phi_{\mathrm{r}} ; k\right)\right|^{2} d \Omega}}$

where the integration is over all points in the sphere of radius $R_{\mathrm{r}}$ and $d \Omega=r_{\mathrm{r}}^{2} \sin \theta_{\mathrm{r}} d \theta_{\mathrm{r}} d \phi_{\mathrm{r}} d r_{\mathrm{r}}$, and $S_{D}\left(r_{\mathrm{r}}, \theta_{\mathrm{r}}, \phi_{\mathrm{r}} ; k\right)$ and $S_{R}\left(r_{\mathrm{r}}, \theta_{\mathrm{r}}, \phi_{\mathrm{r}} ; k\right)$ are the desired and reproduced sound fields, respectively.

Since the designed array is non-symmetric unlike a spherical array, we quantify the performance of the loudspeaker system for desired sound fields due to plane waves arriving from all directions in 3D. Figure 6 shows the MSE of the system as a function of the elevation and azimuth angle of an incoming plane wave sound field. As before, the desired reproduction region is a sphere of radius $R_{\mathrm{r}}=11 \mathrm{~cm}$ and the frequency of the plane wave is $500 \mathrm{~Hz}$. Observe (from Fig. 6) that MSE varies for different elevation of the plane wave and relatively constant over azimuth. This is due to the fact that the loudspeaker array is non symmetric with elevation and symmetric in azimuth, or in other terms symmetric with respect to the z-axis. Theoretically, we have shown in Section VI-B that there are errors outside the reproduction region due to aliasing. Figure 7 depicts MSE as a function of the radius $R_{\mathrm{r}}$ of the reproduction region for different plane wave sound fields. Upon observing it, we see that MSE for a spherical region of radius $11 \mathrm{~cm}$ is quite small, showing that a third order system does accurate reproduction. However, outside of this region, with increasing radius, the error increases as higher order harmonics $(>3)$ are included in the desired field which are not accounted for in the calculation of aperture coefficients.

\section{Aliasing Errors/ Broadband performance}

Based on the theory and analysis, for a fixed order system, upon increasing the frequency, the region of interest for accurate sound field reproduction reduces. Figure 8 shows the desired and reproduced fields for a plane wave at $f=2500$ $\mathrm{Hz}$ arriving from two different angles of $(\theta, \phi)=90^{\circ}, 45^{\circ}$ and $(\theta, \phi)=135^{\circ}, 60^{\circ}$ respectively. The reproduced field is

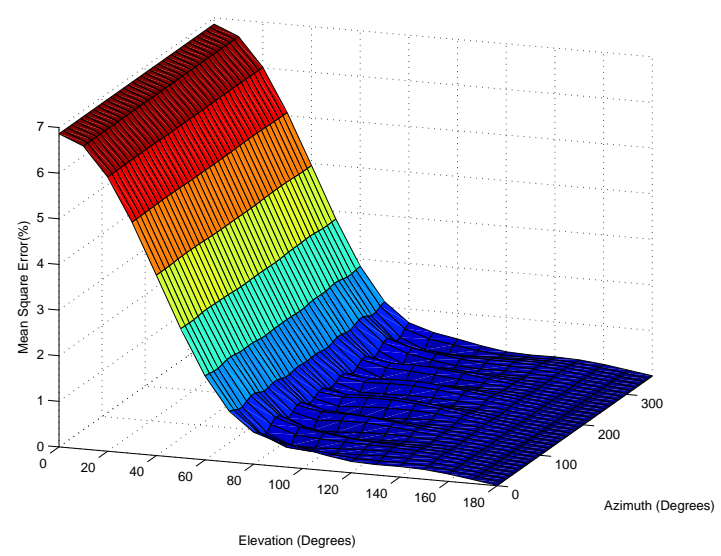

Fig. 6: Mean Square Error (MSE) of the reproduce sound field with a spherical reproduction region of radius $R_{\mathrm{r}}=11 \mathrm{~cm}$ as a function of the elevation and azimuth angle of a incoming plane wave desired sound field of frequency of $500 \mathrm{~Hz}$.

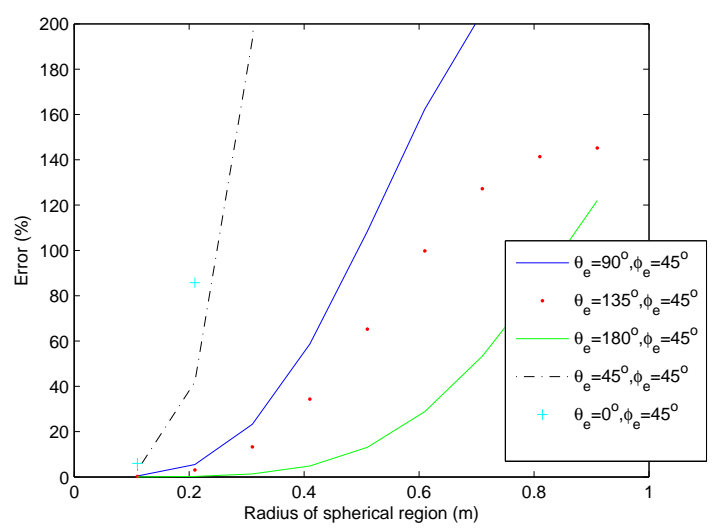

Fig. 7: Error as a function of radius of spherical region of interest for a plane wave operating at $500 \mathrm{~Hz}$ arriving at varying elevation angles and fixed azimuth $\phi=45^{\circ}$ 

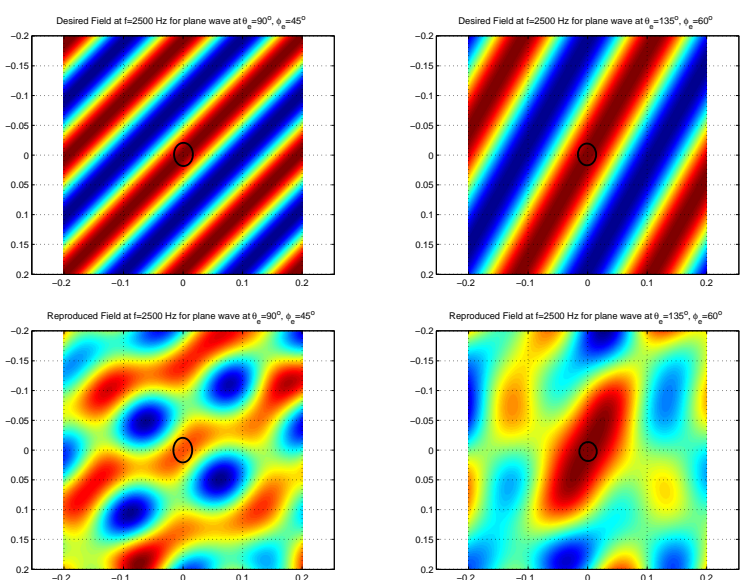

Fig. 8: Real part of the desired and reproduced sound fields: A plane wave arriving at an operating frequency of $2500 \mathrm{~Hz}$ and direction of $(\theta, \phi)=90^{\circ}, 45^{\circ}$ on the left and $(\theta, \phi)=$ $135^{\circ}, 60^{\circ}$ on the right respectively. The encircled region is the truncated region of interest.

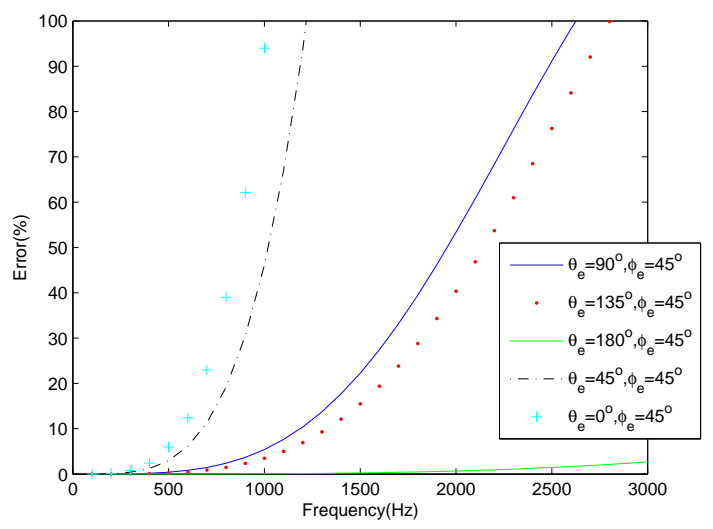

Fig. 9: Error as a function of frequency on a spherical region of $11 \mathrm{~cm}$ for a plane wave arriving over varying elevation angles and fixed azimuth $\phi=45^{\circ}$

accurate in a smaller region but however aliasing errors occur over larger areas.

Figure 9 depicts the MSE over the 3D spherical region of radius $11 \mathrm{~cm}$ for a plane wave as a function of frequency arriving at $\theta=0^{\circ}, 45^{\circ} 90^{\circ}, 135^{\circ}, 180^{\circ}$ and $\phi=45^{\circ}$. From Figure 9, we note that the MSE is low showing accurate reproduction at $f=500 \mathrm{~Hz}$, which is consistent with the design and theory.

\section{CONCLUSION}

Practical implementation of three dimensional sound field reproduction systems in the literature are difficult due to the spherical nature of the loudspeaker array configurations. A practically realizable method of 3 dimensional sound field reproduction system has been proposed in this paper. The methodology uses some properties of the associate Legendre functions to control selected spherical harmonics modes of the sound field by specific modes of the circular loudspeaker driving functions. The loudspeaker configuration consists of number of circular arrays where the radii can be adjusted to fit to a given room size without compromising the design. A third order system using 18 loudspeakers has been implemented and tested for different sound fields. A detailed error analysis has also given with quantifying aliasing errors and broadband performance.

\section{REFERENCES}

[1] F. Fazi, P. Nelson, and R. Potthast, "Analogies and differences between three methods for soundfield reproduction," in Ambisonics Symposium, Graz, Austria, June 2009.

[2] M. A. Gerzon, "Periphony: With-height sound reproduction," J. Audio Eng. Soc., vol. 21, no. 1, pp. 2-10, 1973.

[3] J. Bamford, "An analysis of ambisonic sound systems of first and second order," Ph.D. dissertation, University of Waterloo, Canada, 1995.

[4] R. Elen, "Ambisonics: The surround alternative," in Surround 2001 Conference, Beverly Hills, CA, USA, 2001.

[5] A. D. Blumlein, "Improvements in and relating to sound-transmission, sound-recording and sound-reproducing systems," UK Patent 394325 , 1931.

[6] J. Daniel, R. Nicol, and S. Moreau, "Further investigations of high order ambisonics and wavefield synthesis for holophonic sound imaging," in Proc. 114th AES Convention, Amsterdam, 2003.

[7] J. Daniel and S. Moreau, "Further study of sound field coding with higher order ambisonics," in Proc. AES 116th Convention, 2004.

[8] J. Ahrens and S. Spors, "Analytical driving functions for higher order Ambisonics," in IEEE Int. Conf. on Acoustics, Speech and Sig. Process., 2008, pp. 373-376.

[9] Y. J. Wu and T. D. Abhayapala, "Theory and design of soundfield reproduction using continuous loudspeaker concept," IEEE Trans. Audio, Speech, and Language Proc., vol. 17, no. 1, pp. 107-116, January 2009.

[10] D. B. Ward and T. D. Abhayapala, "Reproduction of a plane-wave sound field using an array of loudspeakers," IEEE Trans. Speech Audio Process., vol. 9, no. 6, pp. 697-707, September 2001.

[11] M. Poletti, "Robust two-dimensional surround sound reproduction for nonuniform loudspeaker layouts," Journal of Audio Engineering Society, vol. 55, no. 7/8, p. 598, 2007.

[12] T. Betlehem and T. D. Abhayapala, "Theory and design of sound field reproduction in reverberant rooms," J. Acoust. Soc. Am., vol. 117, no. 4, pp. 2100-2111, April 2005.

[13] H. Teutsch, Modal Array Signal Processing: Principles and Applications of Acoustic Wavefield Decomposition. Springer, 2007. 
[14] H. Teutsch and W. Kellermann, "Detection and localization of multiple wideband acoustic sources based on wavefield decomposition using spherical apertures," in IEEE International Conference on Acoustics, Speech and Signal Processing (ICASSP), 2008, pp. 5276-5279.

[15] A.J. Berkhout, D. De Vries, and P. Vogel, "Acoustic control by wave field synthesis," J. Acoust. Soc. Am., vol. 93, pp. 2764-2778, May 1993.

[16] D. D. Vries and M. M. Boone, "Wave field synthesis and analysis using array technology," in Proc. 1999 IEEE Workshop on Applications of Signal Processing to Audio and Acoustics, New York, USA, Oct. 1999.

[17] S. Spors, R. Rabenstein, and J. Ahrens, "The Theory of Wave Field Synthesis Revisited," in Proc. 124th AES Convention, Amsterdam, Netherlands, May 2008.

[18] M. Naoe, T. Kimura, Y. Yamakata, and M. Katsumoto, "Performance Evaluation of 3D Sound Field Reproduction System Using a Few Loudspeakers and Wave Field Synthesis," in Universal Communication, 2008. ISUC'08. Second International Symposium on, 2008, pp. 36-41.

[19] N. Epain and E. Friot, "Active control of sound inside a sphere via control of the acoustic pressure at the boundary surface," Journal of Sound and Vibration, vol. 299, no. 3, pp. 587-604, 2007.

[20] M. Poletti, "Three-Dimensional Surround Sound Systems Based on Spherical Harmonics," Journal of Audio Engineering Society, vol. 53, no. 11 , pp. 1004-1025, 2005.

[21] S. Moreau, J. Daniel, and S. Bertet, "3d sound field recording with higher order ambisonics: Objective measurements and validation of a 4 th order spherical microphone," in AES 120th Convention, Paris, France, 2006.

[22] M. Poletti, "A Spherical Harmonic Approach to 3D Surround Sound Systems," Forum Acousticum, pp. 311-317, 2005.

[23] D. Malham and A. Myatt, "3-D sound spatialization using ambisonic techniques," Computer Music Journal, vol. 19, no. 4, pp. 58-70, 1995.

[24] R. Nicol and M. Emerit, "3d-sound reproduction over an extensive listening area, a hybrid method derived from holophony and ambisonic," in AES 16th International Conference, 1999, pp. 436-453.

[25] Z. Li, R. Duraiswami, and N. Gumerov, "Capture and recreation of higher order $3 \mathrm{~d}$ sound fields via reciprocity," in ICAD'04, 2004, pp. 6-9.

[26] D. Excell, "Reproduction of a $3 d$ sound field using an array of loudspeakers," Honours Thesis Australian National University, June 2003.

[27] F. Fazi, P. Nelson, J. Christensen, and J. Seo, "Surround system based on three-dimensional sound field reconstruction," in Proc. 125th AES Convention, 2008, p. 21 Pages.

[28] F. Bowman, Introduction to Bessel Functions. New York: Dover, 1958.

[29] T. D. Abhayapala, T. S. Pollock, and R. A. Kennedy, "Characterization of 3d spatial wireless channels," in IEEE 58th Vehicular Technology Conference, VTC 2003-Fall, vol. 1, 2003, pp. 123-127.

[30] L. Kinsler, A. Frey, A. Coppens, and J. Sanders, Fundamentals of Acoustics. New York: John Wiley \& Sons, 1999.

[31] D. Colton and R. Kress, Inverse Acoustic and Electromagnetic Scattering Theory, 2nd ed., ser. Applied mathematical sciences. New York: Springer, 1998.

[32] T. D. Abhayapala and A. Gupta, "Spherical harmonic analysis of wavefields using multiple circular sensor arrays," IEEE Trans. Audio, Speech, and Language Proc., vol. 18, no. 6, pp. 1655-64, August 2010.

[33] H. Hall and S. Knight, Higher Algebra: a Sequel to Elementary Algebra for Schools. Elibron Classics, 2001.



Thushara D. Abhayapala received the B.E. degree (with honors) in interdisciplinary systems engineering in 1994, and the Ph.D. degree in telecommunications engineering, both from the Australian National University (ANU), Canberra, in 1999. From 1995 to 1997, he was a Research Engineer with the Arthur C. Clarke Center for Modern Technologies, Sri Lanka. Since December 1999, he has been a faculty member with the Research School of Information Sciences and Engineering, ANU. He was the Leader of the Wireless Signal Processing (WSP) Program at the National ICT Australia (NICTA) from November 2005 to June 2007. Currently, he is the head of the school of Engineering at the Australian National University.

His research interests are in the areas of audio and acoustic signal processing, space-time signal processing for wireless communication systems, and array signal processing. He has supervised 24 research students and coauthored over 160 peer-reviewed papers. He is currently an Associate Editor for the EURASIP Journal on Wireless Communications and Networking.

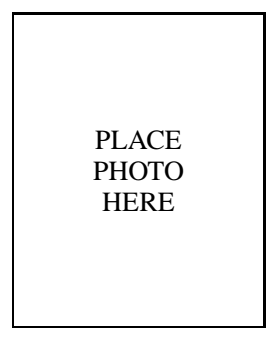

monic analysis.
Aastha Gupta received her B.E. degree in telecommunication and Electronics Engineering with honours from the Australian National University, Canberra Australia, in 2006. She has now completed her PhD with the Applied Signal Processing Group, College of Engineering and Computer Science, at the Australian National University. She is a graduate member of the IEEE and Engineers Australia. Her research interests are in spatial signal processing techniques in acoustic modeling, measurement and reproduction of audio scenes using spherical har- 